In mentioning these causes of failure I may perhaps add - that a little practice and familiarity with the operation goes far to eliminate them, and I see far less of them now than formerly. With these few exceptions I have found the operation entirely satisfactory. The children have nice supple active feet, permanently corrected, and they are from the beginning freed from the burden of instruments.

\section{PNEUMOCOCCAL INFLUENZA.}

\section{BY}

\section{O. F. T. EAST, M.A., B.M., B.CH.Oxon., M.R.C.P.LonD.,} OUT-PATIENT MEDICAL OFFTCER, KING'B COLLEGE HOBPITAL.

THE name "influenza" is applied to a variety of ailments. In the recent great pandemic $B$. influenzae was shown to be the infecting organism in a very large number of cases; there may be, however, small local epidemics, influenzal in type, which are caused by other organisms. Clinically the cases have the appearance of influenza, and one might class them, as Sir William Osler suggested, under the head of "influenza nostras."

The series of cases here described occurred among the nursing staff and students of a hospital during the early part of this year. The epidemic began early in January and went on till March, and comprised some forty cases. The pneumo. coccus was the organism found in overwhelming predominance on examination of throat swabs by direct smear and culture in every case. M. catarrhalis and streptococci also occurred, but were in the minority, and $B$. influenzae was not seen. Pneumococci were also the cause of such complications as occurred. Clinically the cases varied from a slight headache, sore throat, and fever to rery severe prostration.

In almost every case the first symptoms were referred to the upper air passages. Some complained of having had a cold for a day or two, or a sore throat; in others the onset was more sudden. Then followed headache, which was usually frontal, and attacks of shivering, with pains all over the body, especially in the back and limbs. Such symptoms are similar to those described as the nervous symptoms of influenza. The headache in some instances was intense. Most cases had more or less photophobia, and in some there was considerable conjunctival injection. The tongue was coated with white fur as a rule; in four instances there was vomiting, which persisted for three days in one case. There was no marked diarrhoea or constipation. Fever appeared with the other symptoms. The temperature in some cases reached $104^{\circ} \mathrm{F}$; in others there was only a slight rise. As a rule there was in uncomplicated cases no fever after four or five days.

Sore throat was the most common complaint, and there were patches on the tonsils in most cases; injection was acute in all. The throat and nasopharynx seem to have been the points of attack of the infecting organism. In five cases there appeared on the fauces and soft palate, and on the uvula to a varying degree, a whitish, shining exudate, which had a glazed look; it gradually spread over the surrounding parts, was very thin, almost transparent, and easily detach. able. From each of these cases almost pure cultures of pneumococci were obtained. The parts of the throat not covered by the exudate were very injected. The presence or absence of tonsils did not appear to have any influence on the incidence of the infection, but the patients without tonsils got off more lightly. Only three slight cases of adenitis occurred, and the glands at the angle of the jaw were only affected to a small degree in the most severe cases of throat infection. There was not much tendency to downward spread of the infection; bronchitis occurred twice, and there were two cases with pulmonary consolidation; one of the latter ended in widespread bronchopneumonia which proved fatal.

In six of the most severe cases a scarlatiniform erythema appeared early in the illness. It showed itself uniformly on the limbs, trunk, and face at the same time. The extensor surfaces of the limbs tended to be most affected. There was no itching. As the temperature fell the rash faded, leaving the skin rather yellow. There was a very slight powdery desquamation afterwards in two cases. The appearance of the rash was the signal of acute infection, and the patient was soon very ill. The throat was always markedly affected in these cases, and four of them showed the distinctive exudate described above.
Direct spread of the pneumococcus caused such complica. tions as there were. The pus from a double mastoid infec. tion, which set in on the fourth day, gave a pure culture of pneumococci. The same was true of three cases of empyema of the antrum, as also of an acute case of conjunctivitis which began three days after the onset of the original infection.

Clinically these cases exhibited the rather protean sym. ptoms of influenza, although $B$. influenzae was not the cause. The pneumococcus was often present as a secondary infection in cases of true influenza in the recent epidemics, but this outbreak illustrates the observation of Pulfrey that a primary pneumococcal infection may cause an epidemic with all the clinical appearances of true influenza. This has also been noted by Peset," who found the pneumococcus to be the cause in 62 per cent. of cases in an epidemic.

Toxic erythemata are seen in acute infections, generally streptococcal in origin. They may occur in pyaemia ${ }^{1} 8$ Such rashes have been described in cases of influenza by Hainiss $^{2}$ and others. Sir W. Osler mentions them as occurring occasionally in pneumonia. No doubt in the outbreak here described they were caused by the absorption of toxins from the throat; it may be noted that they were seen only in the worst cases. The appearance of the throat exudate described above was very striking and distinctive. It would appear to be characteristic of acute pneumococcal infection in the throat.

These few cases serve to show how closely true influenza may be mimicked by an infection due to another organism. My thanks are due to Dr. Harold Wiltshire for his kindness in allowing me to publish this account.

REFERENCES.

1 Gioseff, M.: Gli Esanteme scarlattiniformi nella grippe, Riforma med., 1920, 46, 604. 2 Hainiss: Scharlachartige Exantheme bei Grippe Wren. klin. Woch., 1919, 32, 201. 3 Leimdorfer, A. : Uber Exantheme und Policlinico Valencia, 1919, 7, 720 . B Pulfrey : New York Med. Journ., 1919, $110,310,368$.

\section{A CASE OF BRONCHO-BILIARY FISTULA.} BY

A. G. YATES, M.A., M.D., M.R.C.P., PHYSICIAN TO THE SHETFIELD ROYAL INFIRMARY.

THE following case is an unasual one, and at the same time of sufficient practical clinical interest to merit its being placed on record.

A labourer, aged 47, went to his doctor in June, 1921, complaining of some loss of weight and of feeling generally weak and ill. ' A few davs later a severe hiccup commenced which lasted for nine days and then ceased abruptly. It was so severe that it deprived him of sleep and serionsly interfered with esting, and even with speaking. On its disappearance he complained of nothing beyond general weakness, but three weeks later a cough began. with copious expectoration which he described as being " like brown jelly and sometimes streaked with blood." This continued for a month. when the colour of the sputum suddenly changed to yellow. It was now intensely irritating and associated with a violent paroxysmal cough which sometimes led to vomiting.

Such was his condition on admission to hospital on November 5th, 1921. His previous record included scarlet fever at 8, pnenmonia at 16, and gonorrboea at 19 . He had never been abroad, and monia at 16 , and gonorrboea at 19 . He had never

He was found on admission to be coughing up large quantities He was found on admission to be coughing up large quantities
of clear bright yellow fluid, which consisted almost entirely of bile. It contained a few Gram positive cocci and one or two cells, but no pus. No traces were found of amoebae or the hooklets of Tatnia echinococcus. He was emaciated and weighed $7 \mathrm{st}$. $43 \mathrm{lb}$. Before his illness he weighed $9 \mathrm{st}$. $2 \mathrm{lb}$. He was not jaundiced.

Examination of the chest revealed a small area of impaired resonance at the base of the right lung over which medium crepitations could be heard. The breath sounds over this area were harsh and vesicular in character; the vocal fremitus and resonance were not altered.

The liver was a little enlarged and just below the right costal margin could be felt somewhat rounded and hard. There were several dilated veins in the abdominal wall, chiefly on the right side both above and below the umbilicus. The spleen was not enlarged, there was no ascites, and examination of the faeces failed to revesl any evidence of amoebae. The Wassermann failed to revesl any evidence of amoebae. The Wassermann reaction was negative and radiograms showed nothing abnormal. It was clear that a communicstion had in some way been effected between the bile passages and the bronchi, and as the condition was causing acute distress Professor A. M. Connell operated on November 25th. Portions of the eighth and ninth ribs were resected on the right side and the upper surface of the diaphragm explered. Dense adhesions were found between the base of the lung and the upper surface of the diaphragm, but no opening through the diaphragm was seen. The adher ent surfaces were 